\title{
Rehabilitation After Gluteus Medius and Minimus Treatment
}

\author{
Victor M. Ilizaliturri Jr., M.D., Rafael Zepeda Mora, M.D., and \\ Liliana Patricia Rodríguez Vega, M.D.
}

\begin{abstract}
We reviewed the current literature regarding rehabilitation after gluteus medius and minimus tears as part of a conservative management or postoperative protocol. The greater trochanteric pain syndrome includes a constellation of pathologies that generate pain in the greater trochanteric region and may be accompanied by varying degrees of hip abductor disfunction. It may be related to tendinitis of the gluteus medius and minimus, greater trochanteric bursitis, or even formal tears of the hip abductor tendons. The initial management strategy of the hip abductor tears is conservative, including different anti-inflammatory therapies such as physical therapy and cortisone and platelet-rich plasma injections. The clearest indication for surgical management is failure of conservative management and loss of abductor muscle power. Surgical management has been performed both open and endoscopic with good reported clinical results. More severe tears typically require a more rigid and complex type of fixation. Exorcise intervention seem to improve symptoms after 4 months to a year of therapy therefore a very close supervision of the rehabilitation protocol is mandatory. Gluteus medius and minimus tears are frequent and may be not diagnosed timely. Treatment of these of lesions is based on the knowledge of pathomechanics involved and the extent of injury to the tendon and muscle tissue. Conservative management is based on protecting the hip abductor tendons from excessive tensile and compression stresses while applying progressive load in conjunction with physical and medical anti-inflammatory measures. Surgical treatment is indicated when conservative management fails or an abductor power deficit is associated with pain. Similar physical therapy protocols to those used in conservative management are used postoperatively. Level of Evidence: Level V, expert opinion.
\end{abstract}

G uteus medius and minimus tears have been identified recently as an important source of lateral hip pain and in some cases gait problems that may be an important cause of disability. Tears of the gluteus medius and minimus are included in what is now recognized as the greater trochanteric pain syndrome (GTPS), which includes a constellation of pathology ranging from greater trochanteric bursitis, tightness of the iliotibial band, and tendinitis to formal

From the National Rehabilitation Institute of Mexico, Mexico City, Mexico. The authors report that they have no conflicts of interest in the authorship and publication of this article. Full ICMJE author disclosure forms are available for this article online, as supplementary material.

Received September 20, 2021; accepted October 26, 2021.

Address correspondence to Victor M. Ilizaliturri Jr., M.D., National Rehabilitation Institute of Mexico, Av. Mexico-Xochimilco 289, Coapa, Arenal Tepepan, Tlalpan 14389, Mexico City, Mexico.E-mail: vichip2002@yahoo. com.mx

(C) 2021 Published by Elsevier Inc. on behalf of the Arthroscopy Association of North America. This is an open access article under the CC BY-NC-ND

license (http://creativecommons.org/licenses/by-nc-nd/4.0/).

2666-061X/211385

https://doi.org/10.1016/j.asmr.2021.10.024 muscle and tendon tears of the hip abductors. ${ }^{1}$ GTPS affects between $10 \%$ and $25 \%$ of the general population, with a peak incidence between the fourth and sixth decades of life. ${ }^{2}$ In a multicenter observational study, Segal et al. ${ }^{3}$ found that the prevalence of GTPS was $15 \%$ for unilateral disease and $8.5 \%$ for bilateral disease in women and $6.6 \%$ for unilateral disease and $1.9 \%$ for bilateral disease in men. The clearest indication for surgical treatment of gluteus medius and or minimus tears seems to be loss of abductor power (Medical Research Council Grading of Power) less than $4^{4}$ and Trendelenburg gait. ${ }^{5}$

Gluteus medius and/or gluteus minimus tendinopathy is now accepted as the most prevalent pathology in those with pain and tenderness over the greater trochanter. ${ }^{6}$

Understanding the pathomechanics of these conditions is mandatory to understand the principals for physical therapy for both conservative management and therapy after surgical treatment. We reviewed current literature regarding rehabilitation after gluteus medius and minimus tears as part of a conservative management or postoperative protocol. 


\section{Pathomechanics of Gluteal Medius and Minimus Tendon Pathology}

Mechanical loading is a strong driver of the biological processes that occur within a tendon and these, in turn, determine its structural shape and load-bearing capacity. A tendon can undergo both catabolic and anabolic processes. Under conditions of normal loading, these processes are balanced and provide the basis for a healthy homeostatic state within the tissue. This balance can be altered by the type, intensity, and frequency of loading. ${ }^{7}$ Tendon loads can be longitudinal (tension or traction loads) or perpendicular (compression) to its collagen fibers. Tensile loads can be applied actively via muscle contraction or passively via stretching. Tendon loads will be greater when the muscle is active and the tendon lengthens at the same time, that is, with eccentric contractions, where anatomical compression may also occur. ${ }^{8}$

The pathomechanics associated with the development of gluteal tendinopathy are like those proposed for other insertional tendinopathies: relatively increased tensile load (overload) ${ }^{9}$ or decreased (protection against stress/load). The combination of traction and compression overload appears to be particularly damaging. ${ }^{10}$ Gradual increases in tensile load with adequate adaptation and recovery time induce a net anabolic effect, with a subsequent increase in tendon load capacity. ${ }^{7}$ A rapid increase in the intensity and/or frequency of the tensile load can lead to failure of adaptation and a net catabolic effect. Net catabolism also results from a lack of tensile and compressive loading. At the cellular level, stress deprivation and compression induce the expression of catabolic enzymes, which degrade type 1 collagen $^{11}$ and increase the expression of large proteoglycans in tenocytes, which cleave collagen fibers. ${ }^{12}$ This matrix degradation and changes in tenocyte expression reduce loadbearing capacity and predispose to injuries with relatively low tensile loads. ${ }^{10}$ The interaction between bone and muscle factors must be considered to understand how compressive load or protection against stress contributes to the underlying pathomechanics of this disorder. ${ }^{13}$

The tendons of gluteus medius and gluteus minimus, can be compressed by the iliotibial band at its insertion into the greater trochanter. Compressive load is influenced by the position of the hip joint. Compressive loads in this region of 4 Newtons $(\mathrm{N})$ at $0^{\circ}$ hip adduction have been shown to rise to $36 \mathrm{~N}$ at $10^{\circ}$ adduction and $106 \mathrm{~N}$ at $40^{\circ}$ adduction. ${ }^{14}$ During activities with excessive hip adduction, compressive loads are likely to accumulate on the tendons, both in static postures and in dynamic tasks. ${ }^{15}$ It is likely that during activities such as standing with one hip adducted, sitting with the knees together or crossed in adduction, an excessive tilt or lateral displacement of the pelvis during dynamic single-leg loading tasks, and running in a pattern gait with foot-ground contact with excessive hip adduction, compressive loads accumulate on the tendons.

Greater ranges of hip flexion also can alter iliotibial band tension due to the significant fascial confluence of the iliotibial band with the gluteal fascia and lumbodorsal fascia, ${ }^{16}$ thus potentially contributing to compression of the gluteus medius and gluteus minimus tendons. The combination of greater ranges of hip flexion and adduction is likely to induce a substantial compressive loading on the gluteus medius tendon.

The abductor mechanism of the hip involves 2 muscle group synergies, the first of which is the trochanteric abductor muscles consisting of the gluteus medius and minimus and, second, the tensor fascia lata and the gluteus maximus in conjunction with the vastus lateralis that provide tension to the iliotibial band. ${ }^{15}$ The trochanteric abductors provide $70 \%$ of the abductor force required for pelvic control in a single leg stance, with the remaining $30 \%$ provided by the iliotibial band tensing muscles. ${ }^{17}$ The tensor fascia lata has been shown to hypertrophy and the gluteus medius and minimus to atrophy in those patients with gluteal tendon pathology. ${ }^{18,19}$ The imbalance in the activity of the trochanteric abductors and iliotibial band tensors could alter the relative contribution of these muscle groups in the control of movement or postures in the frontal plane. ${ }^{16}$

Bone morphology also influences the compression forces exerted on the hip by the iliotibial band. At a femoral neck-shaft angle of $128^{\circ}$, the iliotibial band exerts a compression force of around $656 \mathrm{~N}$ on the greater trochanter, but at $115^{\circ}$, the compressive force increases up to $997 \mathrm{~N} .^{14,15}$

The gluteus medius in women has been found to have a smaller insertion on the femur across which to dissipate the tensile load and a shorter moment arm, resulting in reduced mechanical efficiency. This mechanical disadvantage is further accentuated in those with a smaller femoral neck-shaft angle, which may lead to greater tensile loads in female gluteal tendons. ${ }^{20}$ Women who have a less efficient gluteus medius more often use greater adduction during functional activities to provide a mechanical advantage to their abductors. Hip abductors have been shown to generate the greatest forces from a hip adducted position, probably associated with length-tension relationships. ${ }^{21}$ In addition, pretensioning the iliotibial band in adduction provides an advantage for the superficial abductor system, exerting its force through the iliotibial band. These strategies can reduce the tensile load and increase the compressive load in the deeper regions of the gluteus medius and minimus tendons. 


\section{Conservative Treatment of Gluteus Medius and Minimus Pathology}

Initial treatment of abductor tendon pathology is conservative and may include short-term use of nonsteroidal anti-inflammatory drugs, activity modification, physical therapy, local injections of corticosteroid or platelet-rich plasma (PRP), and shock wave therapy. If conservative treatment fails to alleviate symptoms, surgical treatment can be attempted. However, the limited availability of high-quality studies and elusive evidence between tendinosis and partial tears cannot provide definitive conclusions regarding the best treatment of gluteal tendinopathy. Randomized controlled trials are needed to test the proposed treatment modalities. ${ }^{22,23}$

Cortisone is one of the most commonly prescribed treatment methods for gluteal tendinopathy. ${ }^{24,25}$ Cortisone infiltration of the peritrochanteric region, both blind and guided by ultrasound, has been used. However, most studies are case series without controls and studies with sufficient statistical power are scarce. In addition, abductor tendon pathology is rarely confirmed by magnetic resonance imaging. Corticosteroid injection generally provides immediate substantial pain relief within the first month; however, pain is not completely relieved, with a positive response in less than one-half of patients at mid- to long term. ${ }^{26}$ In different series, corticosteroid injection provided moderate pain relief at 4 weeks in $72 \%$ to $75 \%$ of patients, decreasing to $41 \%$ to $55 \%$ at 3 to 4 months ${ }^{25-27}$ and at 12 months, there was no difference in outcomes between the groups receiving corticosteroid injection and those receiving usual care (pain relievers as needed). ${ }^{28}$ One of the drawbacks of corticosteroid injections is the unknown mechanism of action and safety with respect to repeated use. The main mode of action is probably as a local analgesic and not as an anti-inflammatory, interacting with local neuropeptides and neurotransmitters. Recurrence of pain after injection generally reflects the inability of corticosteroids to address the underlying pathology. ${ }^{23}$

Data on the use of PRP to treat gluteal tendinopathy is limited. Mautner et al. ${ }^{29}$ Performed ultrasound-guided PRP injections in 16 patients with chronic gluteus medius tendinopathy. They reported $82 \%$ moderateto-complete lateral thigh pain resolution at 6 months. In a case series study, ultrasound-guided PRP injections in 21 patients with tendinosis or partial tendon rupture without atrophy improved symptoms and functionality at a mean of 20 months of follow-up. ${ }^{30}$ In a randomized clinical trial comparing ultrasound-guided triamcinolone and PRP in 20 patients with major trochanteric pain syndrome, triamcinolone had better initial results but was not superior to PRP at 2 months. ${ }^{31}$ In a prospective randomized control trial investigating only partial tears, ultrasound-guided injection of PRP was superior to cortisone in 70 patients at 12 weeks using the modified Harris Hip Score scale. ${ }^{32}$

Radial shock wave therapy is an alternative treatment modality for gluteal tendinopathy. Shock waves can penetrate soft tissues up to $4 \mathrm{~cm}$, providing both analgesia and healing of the abductor tendons. ${ }^{33}$ The benefit of shock wave therapy in the mid- and long term for gluteal tendinopathy has been reported in the literature. A study published by Rompe et al., ${ }^{25}$ in 2009 reported that at 4 weeks only $13 \%$ of the participants reported an improvement, which increased to $68 \%$ at 4 months and $74 \%$ at 15 months. An additional study by the same group compared the shock wave with several other traditional nonsurgical measures that were not described. The results of this study suggested that a single shock wave therapy treatment would be more effective than other conservative measures at a 12month follow-up. ${ }^{34}$

\section{Postural Hygiene and Exercise}

\section{Postural Hygiene}

Minimizing positions or activities that involve sustained or repetitive compression of the hip abductor tendons tendon has shown to help improve symptomatology, especially when compressive forces are applied in combination with high tensile loads. ${ }^{8,9}$ It is recommended to avoid hip adduction positions, standing cross-legged, and sitting with the knees crossed or together $^{15}$ (Fig 1).

Decubitus postures: In side-lying, the gluteal tendons on both sides are compressed: the lower side against the bed and the upper side due to the position of the hip in adduction (Fig 2). Alternative or modified positions would include lying supine with a pillow under the knees if necessary (to unload the anterolateral hips and lumbar spine). Some patients also can obtain relief in a position that is one-quarter of the prone position, in which the body weight rests on the anterolateral thigh (eliminating the compressive load of the greater trochanter), with the hip higher in relative abduction. ${ }^{15}$

Stretching of the hip adductors, flexors, or extensors combines strong passive tensile loads and compressive loads and should therefore be avoided. Massage and puncture techniques can be used instead of stretching, although strong "iliotibial band releases" can be challenging, as the iliotibial band is often sensitive. ${ }^{3}$ Movement patterns employed during functional weight-bearing tasks and deficiencies observed and used to direct treatment should be assessed. ${ }^{15}$

Controlling high tensile loads, particularly rapid increases in activities that involve an additional stretchshortening or compression cycle, may be critical for 

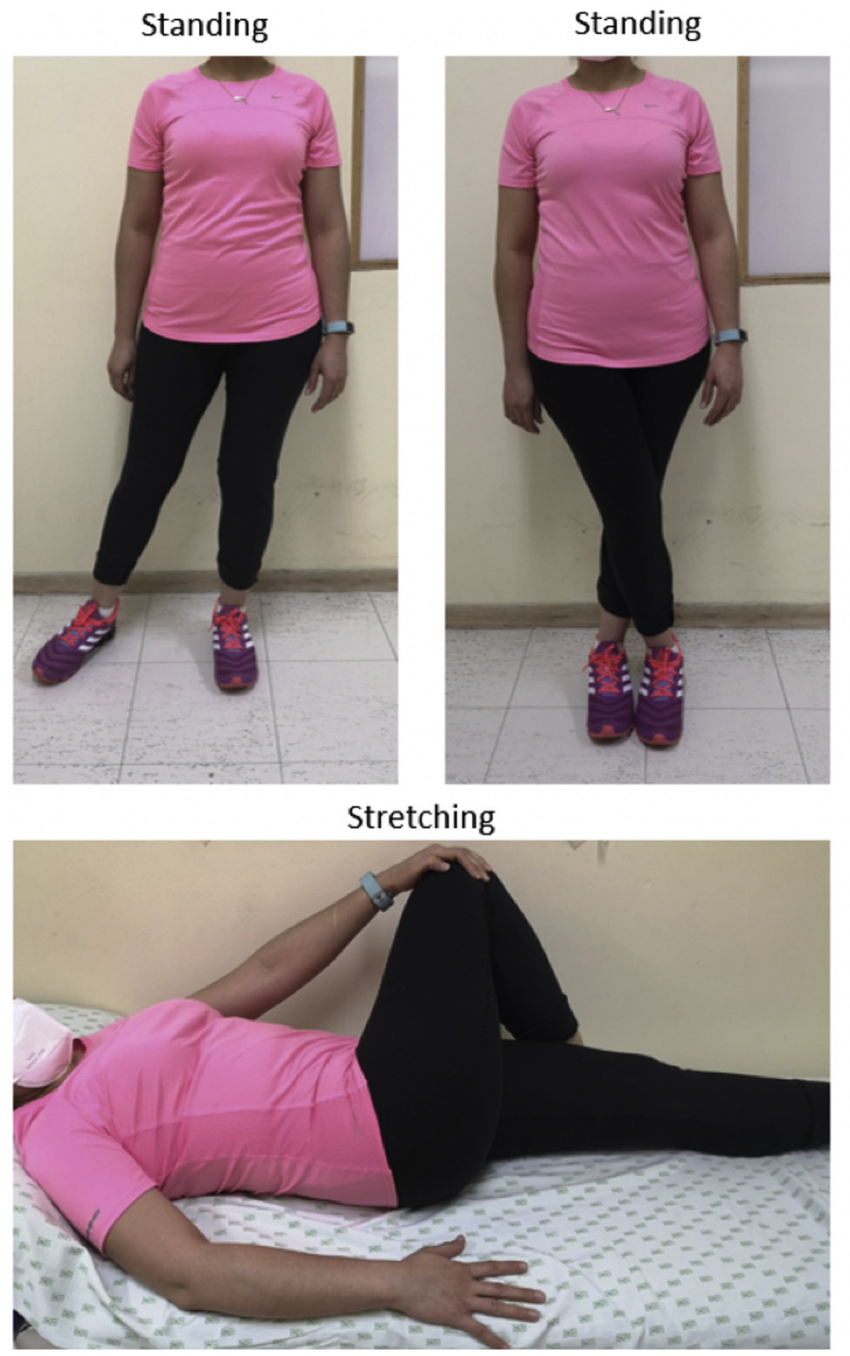

optimal results in people with tendon pain. Patient education regarding avoiding potentially aggravating activities and careful titration of exercise volume are key components of a load management strategy. ${ }^{15}$

Recreational or sporting activity can generally be maintained if the more challenging aspects of those activities are avoided or minimized. Managing the load during activity for the elderly or physically unconditioned patient may involve minimizing climbing hills and stairs and titrating walking distance as necessary to control symptoms. For the athlete, the temporary suspension of long-distance running, tempo running, hill running, and plyometrics may be necessary. Alternative activities such as aquatic exercise and cycling could be explored. As pain subsides and depending on the physical conditioning and occupational and sporting requirements of the patient, hip adduction control can be retrained under higher loads, at faster speeds, and during more complex actions such as running, land and change direction. ${ }^{15}$
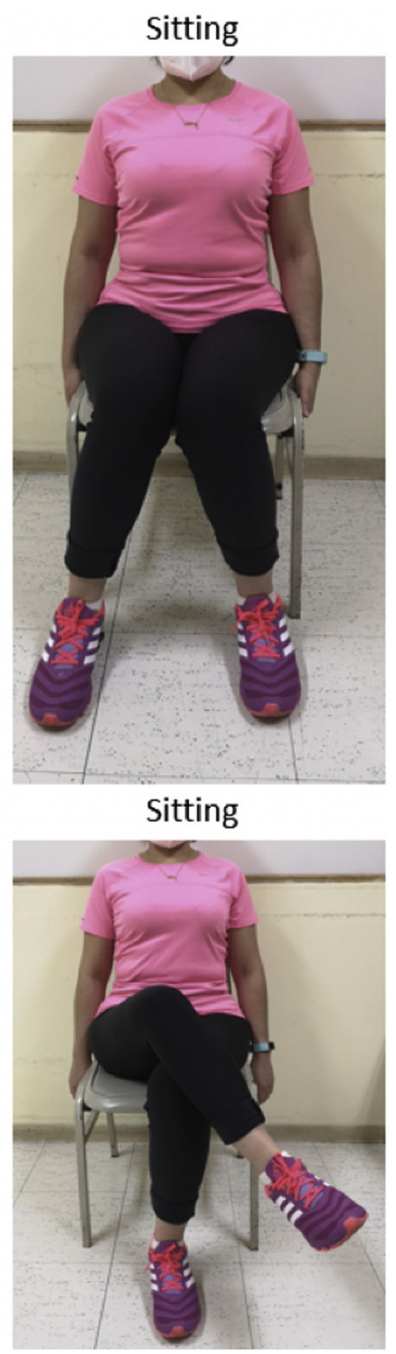

Figure 1. Clinical photographs: Standing, top left, demonstrates a relaxing position of the iliotibial band. Top center, leg-crossing increases tension on the iliotibial band. Siting, top right, this position will relax the iliotibial band. Bottom right, leg-crossing sitting, this will increase pressure on the iliotibial band. Bottom left, supine stretching of the iliotibial band.

\section{Exercise}

The implementation of an early and gradually progressive tensile loading program (in minimally adducted hip positions) aims to reduce pain and improve tendon capacity. ${ }^{8}$ In addition, strengthening exercises along with specific exercises in functional movements, at graded levels of difficulty appropriate to the individual, are likely to be key to rehabilitation.

\section{Isometric Exercises}

Sustained isometric muscle contractions are commonly used clinically for the management of tendon pain due to their analgesic effects. ${ }^{8,35,36}$ Lowintensity sustained contractions (25\% maximal voluntary isometric contraction) are more effective in raising pain thresholds than high intensity $(80 \%)$ in the normal population. ${ }^{37}$

The optimal isometric loading dose is yet to be determined for tendon pain and may vary with the characteristics of the patient and with the particular 


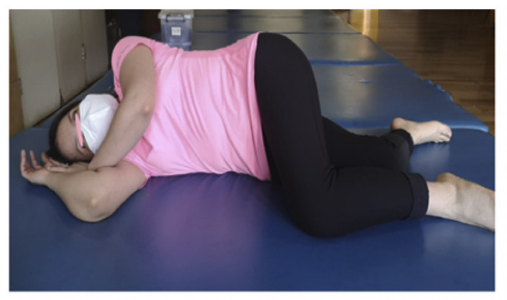

Sidelying

Lowermost hip: weight directly

over greater trochanter.

Uppermost hip: flexed and

adducted.

High Compression

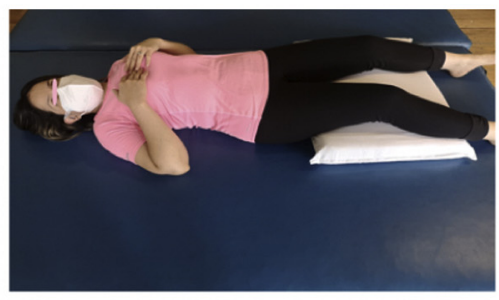

Supine

Hips slightly abducted.

No Compression

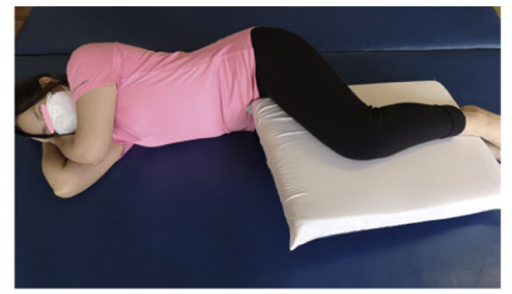

Modified Sidelying

Pillows between legs.

Reduced Compression

Figure 2. This photographs demonstrate positions of high and low compression of the iliotibial band supine and sidelying.

tendon and its anatomical relationships. A lowintensity effort focused on recruitment of the trochanteric abductor and thus loading these tendons in a painless manner is recommended for patients with painful gluteal tendinopathy. ${ }^{15}$ Greater isometric loads, with at least slight hip abduction to avoid compression, may be possible once the patient's response is carefully evaluated.

A recently published systematic review studied gluteus medius and minimus activity levels measured with surface electromyography using the percentage of maximal isometric voluntary contraction. Through this review, a group of exercises could be classified according to the percentage of isometric activity generated in the abductor muscles ${ }^{38}$ (Table 1).

Low-load, low-velocity isometric hip abduction may be performed on the side, with the affected side up, and pillows are used to keep the hip in a neutral position or slightly abducted to avoid compression of the tendon. For bilateral conditions, a slightly abducted supine position can be substituted with a belt or elastic band around the distal thighs for slight resistance. Low-load isometric abduction also may be performed standing with slight abduction and even leaning with the back against a wall or hands on a bench in front, if the patient cannot achieve relaxation of the iliotibial band tensors in an initial position. It is suggested to instruct the patient to slowly increase the intensity of the contraction and to minimize pain in the early stages, until the therapist and the patient have determined how reactive the tendon is ${ }^{15}$ (Fig 3).

To achieve muscle hypertrophy of the gluteus medius and minimus, and to improve the tensile load-bearing of the gluteal tendons, a higher-level tensile loading is required. Low velocity, high-tensile load exercise, typical of muscle hypertrophy programs, has also been shown to produce beneficial effects on tendon structure that are not provided by exclusively eccentric programs. ${ }^{39}$ Targeted strengthening of the trochanteric abductors is perhaps best achieved in people with lateral hip pain by low-velocity, high-tensile load abduction, which minimizes tendon compression. ${ }^{15}$

High-tensile load-bearing exercise should only be performed 3 times per week, allowing adequate time for soft tissue recovery and adaptation. ${ }^{7}$ To achieve muscle hypertrophy, the patient must work at an adequate intensity, although there is considerable potential for exacerbation of pain and even rupture of a weakened degenerative tendon if the traction load is initiated at an excessive level or the load progresses too rapidly. It is safest to start with a moderate level of effort and few repetitions until the tendon response to the tensile load is established. For gluteal tendinopathy, the change in nighttime pain is often a good indicator of response to the exercise program. Once each level of tensile load is well tolerated, the load should be slowly increased and the response monitored to maximize structural change in the musculotendinous unit, while avoiding or minimizing exacerbation of pain. ${ }^{15}$

Management of associated modifiable risk factors and comorbidities is often a feature of gluteal tendinopathy rehabilitation. Although bone morphology cannot be modified, interventions may be necessary to improve lumbar spine, hip, and knee function to optimize control of hip and pelvic motion and thus the loading environment of the gluteal tendons. ${ }^{15}$ Coexisting degenerative joint disease of the lumbar spine, hip, and knee can result in associated weakness of the hip and knee extensors. ${ }^{3,40,41}$ Functional exercises, such as bridge, squat, and step exercises, can serve multiple purposes to optimize control of functional hip adduction, improve function of the lower-extremity extensors, and improve muscle support in the lumbopelvic region, hip and knee. Manual therapy and other specific exercises for the lumbar spine, hip, or knee joint may be required to address coexisting joint disease, but it is important that the principles of respecting gluteal tendon loading, particularly the gluteal tendon, are 
Table 1. Segmental Mean Gluteus Medius Activity Levels (\% MVIC) for Exercises

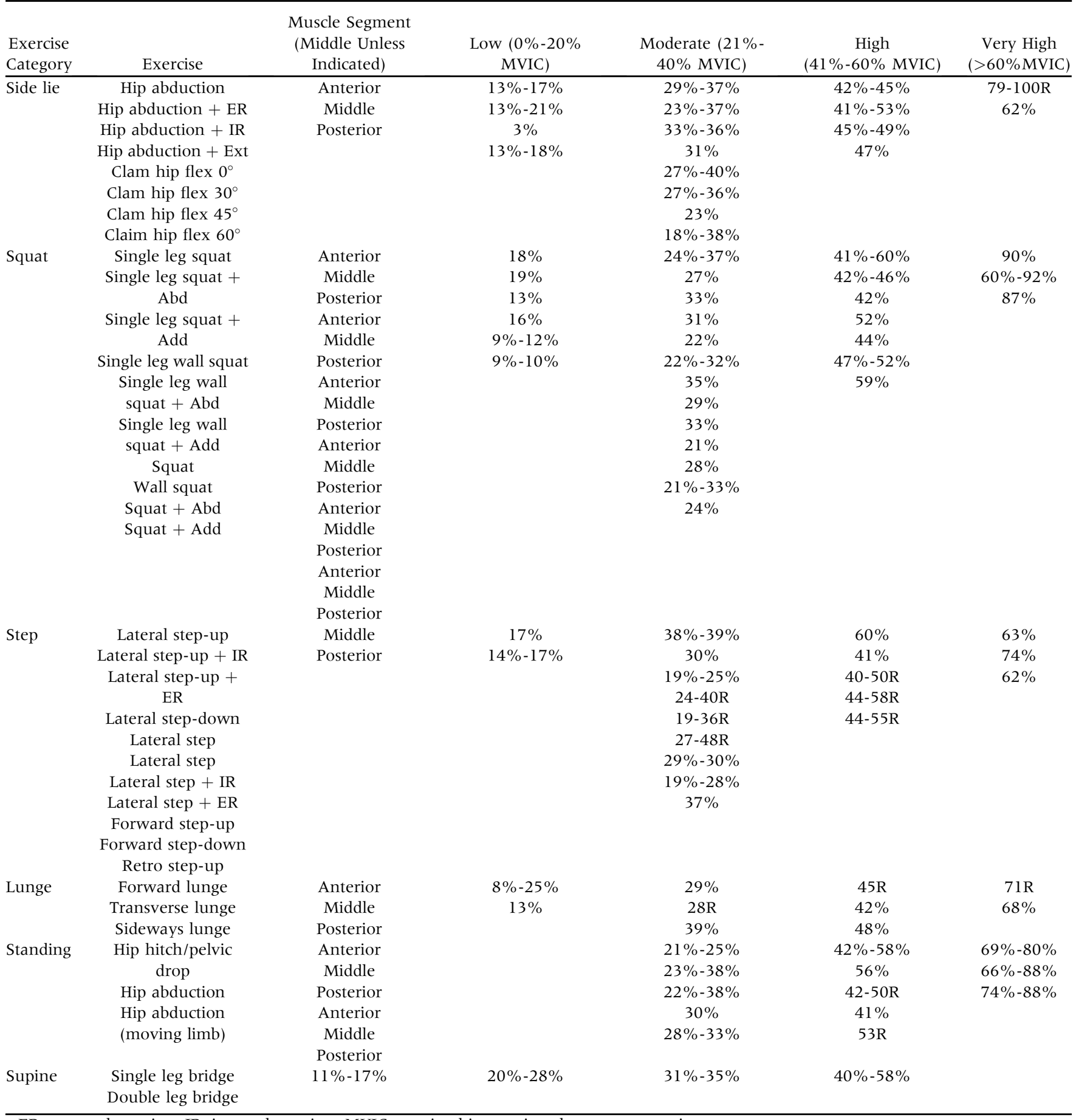

ER, external rotation; IR, internal rotation; MVIC, maximal isometric voluntary contraction.

preserved. compression control. Exercise and general increases in activity also can reduce weight. ${ }^{15}$

\section{Evidence-Based Exercise}

A study examined the effect of an exercise intervention for patients with pain and tenderness on the greater trochanter and positive results in clinical test.
This nonrandomized trial compared exercise at home with shock-wave therapy and corticosteroid injection. In the short term, the exercise intervention was ineffective, as only $7 \%$ of the participants reported an improvement at 4 weeks. However, positive results increased to $40 \%$ at 4 months and $80 \%$ at 15 months. The exercise program included piriformis and iliotibial 


\section{Low-load isometric abduction}

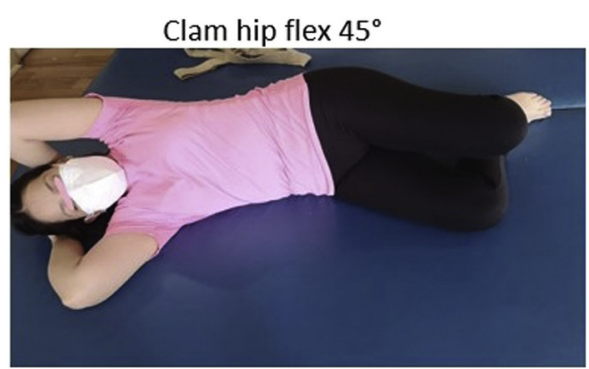

Standing

Figure 3. Hip abductor exercises for the management of gluteal tendinopathy.

\section{Moderate-load isometric abduction}

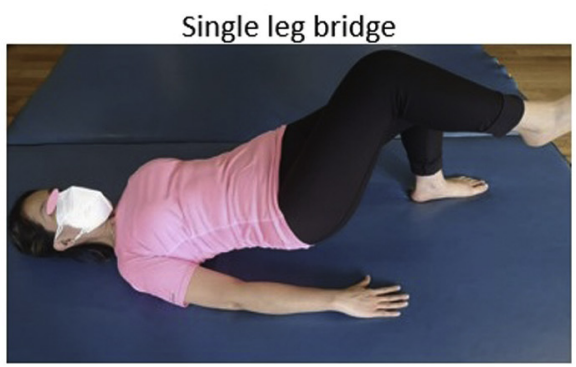

Squat
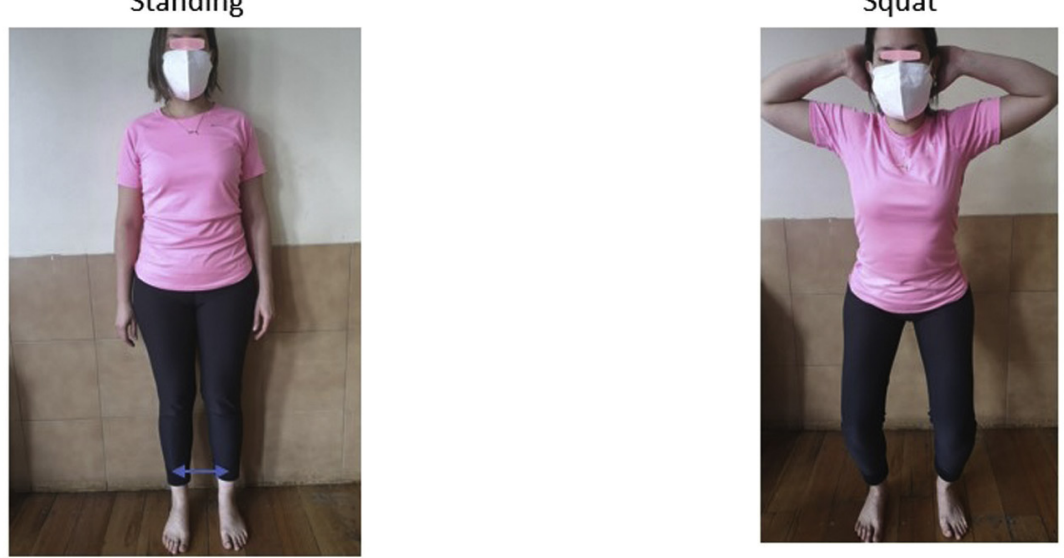

band stretches, potentially exposing the gluteal tendons to compression, strengthening with straight leg raises, wall squats, and prone hip extension, but muscle strengthening of hip abductors was not included. ${ }^{25}$

A prospective, randomized, single-blind, 3-arm clinical trial compared education plus exercise versus the use of corticosteroid injections versus a wait-and-see approach to pain and overall improvement in gluteal tendinopathy. At 52 weeks, the $78.6 \%$ success rate for overall improvement for education plus exercise was better than the $58.3 \%$ success rate for the use of corticosteroid injections (risk difference 20.4\%; $95 \%$ confidence interval $4.9 \%-35.9 \%$ ) and better than the $51.9 \%$ success rate for the wait and see approach (26.8\%; 95\% confidence interval $11.3 \%-42.3 \%)$. In contrast to the study by Rompe et al., ${ }^{25}$ in this study of greater methodologic quality, the benefit of education plus exercise over the injection of corticosteroids was greater in all short-term primary outcomes (8 weeks). This greater rate could be due to the fact that the study focused on the principles of tendon load management and specifically focused on the function of the gluteus medius and minimus muscles with the prescribed exercise program. Exercises at home in the previous study included exercises that did not specifically target these muscles, or control the degree of tendon loading (especially compression loading) for the gluteal musculotendinous complex, and involved limited exercise supervision. $^{42}$

\section{Postoperative Rehabilitation Protocol}

In the case of postoperative rehabilitation protocols, it is of the utmost importance to know the surgical approach, surgical findings, and repair techniques for gluteal medius and/or minimus muscle tears. The rehabilitation protocol will then be adjusted accordingly to offer the greatest benefit and optimize the patient's function and quality of life. The most important considerations are the restriction of mobility and weight bearing, the use of bracing to limit abduction or not, and the allowed ambulation and strengthening exercises.

The Milwaukee Classification of hip abductor tears is a 4-tier system based on the hours on a clock-face. For a right hip the clock hours are assigned from 11 to 3 o'clock and for a left hip the hours are assigned from 9 to $1 o^{\prime}$ clock. Tears will begin at the central fibers of the gluteus medius tendon which corresponds to 1 o'clock in a right hip or 11 o'clock on a left hip. The tears typically extend inferiorly and posteriorly with the grade increasing as the tear progresses. Grade I tears will include the surface extension of one hour in the clock-face, grade II tears will include the surface 
extension of 2 hours, grade III 3 hours, and grade IV almost a complete detachment. ${ }^{43}$

Davies and Davies ${ }^{43}$ reported that there was no statistically significant difference in the results of open surgical technique in 23 open repairs regardless of the type of tear. The majority of the patients were satisfied with the surgical outcome. The mechanical strength of the repair was increased in this series according to the type of rupture. For grade I and II tears, it was sufficient to use $6.5-\mathrm{mm}$ anchors with number 2 sutures, whereas for Grade III and IV bone tunnels and number 5 sutures were necessary. Davies and Davies ${ }^{43}$ suggest that the patients begin with $25 \%$ weight-bearing for the first 6 weeks when the tear was grade I, 8 weeks for grade II, 10 weeks for grade III and 12 weeks for grade IV.

For an open repair of an acute traumatic tear of gluteus medius and minimus tendons, Stanton et al. ${ }^{44}$ decided to restrict the weight-bearing for 12 weeks, with gradual progression. The hip flexion was limited to $90^{\circ}$ for the first 6 weeks and the hip abduction or passive hip internal rotation was allowed after 8 weeks.

For endoscopic management of gluteus medius tendon tears, according to Thaunat et al., ${ }^{45}$ active rehabilitation should be avoided for the first 6 weeks. This consists of avoiding the active lateral rotation and adduction but allowing up to $90^{\circ}$ of hip flexion for the first 3 weeks. An abduction brace should be worn during this period. At 6 weeks, weight-bearing and active lateral rotation and abduction are progressively resumed. ${ }^{49}$

Kocaoglu et al. ${ }^{46}$ and Saltzman et al. ${ }^{47}$ standardized a 3-phase protocol. Phase 1 (0-6 weeks) avoided weightbearing and promoted a gentle passive range of motion and full-time bracing to limit abduction. For Phase 2 (612 weeks), the patients progressed to weight-bearing, the brace was discontinued, and hip-strengthening exercise was initiated. For Phase 3 (>12 weeks), patients progressed to ambulation without assistance and return to general activity.

\section{Discussion}

GTPS is a very common clinical condition that may affect up to $25 \%$ of the general population and is far more prevalent in women. ${ }^{2}$ This condition may range from tendinitis or bursitis to a tear of the gluteus medius and/or minimus tendons. ${ }^{1,3}$ Interest in the diagnosis and management of gluteus medius and minimus tears has increased recently. Management of these tears has been described as both conservative and operative, with the clearest indication for surgical treatment being loss of abductor power and failure of conservative therapy. ${ }^{5}$ Either way, rehabilitation is a very important aspect of the comprehensive management of gluteus medius and minimus tears. Postural hygiene is an important part of rehabilitation management of hip abductor tears; one must minimize positions or activities that involve sustained or repetitive compression of the hip abductor tendons in combination with high tensile loads. It is recommended to avoid hip adduction positions, standing cross-legged, and sitting with the knees crossed or together. ${ }^{8,9,15}$ Abductor hip exercises may not produce early improvement of symptoms but have shown to improve pain and strength at 4 to 12 months after establishing the treatment protocol, this results in the necessity of adequate and close supervision of the patients who undergo physical therapy for hip abductor tears. ${ }^{25}$ The same may be true for exercise protocols after gluteus medius and minimus surgical repair. ${ }^{45-47}$ In the case of postoperative rehabilitation management, the severity of the lesion on the abductor tendons seems not to be related with the clinical outcome but does determine the strength of the mechanical fixation required to fix the tears. Larger and more complex tears require more solid and stronger fixation. ${ }^{43}$

\section{Limitations}

There is limited information in the literature regarding rehabilitation management of gluteus medius and minimus tears. Most of the available information comes from Level III and IV studies reporting on results of case series. This is not a systematic review of the literature but only an analysis of available information and an expert opinion.

\section{Conclusions}

Gluteus medius and minimus tears are frequent and may be not diagnosed timely. Treatment of these of lesions is based on the knowledge of the pathomechanics involved and the extent of the injury to the tendon and muscle tissue. Conservative treatment is based on avoiding postures that may produce tension or compression over the hip abductor tendons while gradually implementing progressive tensile load paired with anti-inflammatory physical therapy. Injections with corticosteroids and PRP or shock-wave therapy may complement physical therapy but should not be considered a standalone form of treatment. In general, failure of conservative treatment and pain associated with abductor power deficit are indications for surgical treatment. There seems to be no difference in the outcome between open or endoscopic management of hip abductor tendon tears and the rigidity of the fixation of the tears depend on the extent of the tear itself. Postoperative physical therapy is similar to that used for conservative management.

\section{References}

1. Kay J, Memon M, Linder D, Randelli F, Ayeni OR. Arthroscopic management of greater trochanteric pain syndrome and abductor tears has demonstrated promising results in terms of improvement in pain scores and 
functional outcomes: A scoping review. Knee Surg Sports Traumatol Arthrosc 2021;29:2401-2407.

2. Poultsides L, Kelly BT. Hip arthroscopy-disorders of the trochanteric space-bursitis and abductor tears. In: Clohisy JC, Beaulé PE, Della Valle CJ, Callaghan JJ, Rosenberg AG, Rubash HE, eds. The adult hip, hip preservation surgery. Philadelphia: Wolters Kluwer, 2015;326-341.

3. Segal NA, Felson DT, Torner JC, et al. Greater trochanteric pain syndrome: Epidemiology and associated factors. Arch Phys Med Rehabil 2007;88:988-992.

4. James MA. Use of the medical research council muscle strength grading system in the upper extremity. $J$ Hand Surg Am 2007;4:154-156.

5. Chandrasekaran S, Vemula SP, Gui C, Suarez-Ahedo C, Lodhia P, Bomb BG. Clinical features that predict the need for operative intervention in gluteus medius tears. Orthop J Sports Med 2015;3:1-5.

6. Long SS, Surrey DE, Nazarian LN. Sonography of greater trochanteric pain syndrome and the rarity of primary bursitis. AJR Am J Roentgenol 2013;201:1083-1086.

7. Magnusson SP, Langberg $H$, Kjaer M. The pathogenesis of tendinopathy: Balancing the response to loading. Nat Rev Rheumatol 2010;6:262-268.

8. Cook JL, Purdam CR. The challenge of managing tendinopathy in competing athletes. Br J Sports Med 2014;48: 506-509.

9. Cook JL, Purdam C. Is compressive load a factor in the development of tendinopathy? Br J Sports Med 2012;46: 163-168.

10. Almekinders LC, Weinhold PS, Maffulli N. Compression etiology in tendinopathy. Clin Sports Med 2003;22: 703-710.

11. Thornton GM, Shao X, Chung M, et al. Changes in mechanical loading lead to tendon-specific alterations in MMP and tIMP expression: Influence of stress deprivation and intermittent cyclic hydrostatic compression on rat supraspinatus and Achilles tendons. Br J Sports Med 2010;44:698-703.

12. Vogel KG, Ordog A, Pogany G, Oláh J. Proteoglycans in the compressed region of human tibialis posterior tendon and in ligaments. J Orthop Res 1993;11:68-77.

13. Grimaldi A, Mellor R, Hodges P, Bennell K, Wajswelner H, Vicenzino B. Gluteal tendinopathy: A review of mechanisms, assessment and management. Sports Med 2015;45: 1107-1119.

14. Birnbaum K, Siebert CH, Pandorf T, Schopphoff E, Prescher A, Niethard FU. Anatomical and biomechanical investigations of the iliotibial tract. Surg Radiol Anat 2004;26:433-446.

15. Grimaldi A, Fearon A. Gluteal tendinopathy: Integrating pathomechanics and clinical features in its management. J Orthop Sports Phys Ther 2015;45:910-922.

16. Stecco A, Gilliar W, Hill R, Fullerton B, Stecco C. The anatomical and functional relation between gluteus maximus and fascia lata. J Bodyw Mov Ther 2013;17: 512-517.
17. Kummer B. Is the Pauwels' theory of hip biomechanics still valid? A critical analysis, based on modern methods. Ann Anat 1993;175:203-210.

18. Sutter R, Kalberer F, Binkert CA, Graf N, Pfirrmann CWA, Gutzeit A. Abductor tendon tears are associated with hypertrophy of the tensor fasciae latae muscle. Skel Radiol 2013;42:627-633.

19. Pfirrmann CW, Notzli HP, Dora C, Hodler J, Zanetti M. Abductor tendons and muscles assessed at MR imaging after total hip arthroplasty in asymptomatic and symptomatic patients. Radiology 2005;235:969-976.

20. Woyski D, Olinger A, Wright B. Smaller insertion area and inefficient mechanics of the gluteus medius in females. Surg Radiol Anat 2013;35:713-719.

21. Kumagai M, Shiba N, Higuchi F, Nishimura H, Inoue A. Functional evaluation of hip abductor muscles with use of magnetic resonance imaging. J Orthop Res 1997;15: 888-893.

22. Barratt PA, Brookes N, Newson A. Conservative treatments for greater trochanteric pain syndrome: A systematic review. Br J Sports Med 2017;51:97-104.

23. Kenanidis E, Kyriakopoulos G, Kaila R, Christofilopoulos P. Lesions of the abductors in the hip. EFORT Open Rev 2020;5:464-476.

24. Lustenberger DP, Ng VY, Best TM, Ellis TJ. Efficacy of treatment of trochanteric bursitis: A systematic review. Clin J Sport Med $2011 ; 21: 447-453$.

25. Rompe JD, Segal NA, Cacchio A, Furia JP, Morral A, Maffulli N. Home training, local corticosteroid injection, or radial shock wave therapy for greater trochanter pain syndrome. Am J Sports Med 2009;37: 1981- 1990.

26. Labrosse JM, Cardinal É, Leduc BE, et al. Effectiveness of ultrasound-guided corticosteroid injection for the treatment of gluteus medius tendinopathy. AJR Am J Roentgenol 2010;194:202-206.

27. Stephens MB, Beutler AI, O'Connor FG. Musculoskeletal injections: A review of the evidence. Am Fam Physician 2008;78:971-976.

28. Brinks A, van Rijn RM, Willemsen SP, et al. Corticosteroid injections for greater trochanteric pain syndrome: A randomized controlled trial in primary care. Ann Fam Med $2011 ; 9: 226-234$.

29. Mautner K, Colberg RE, Malanga G, et al. Outcomes after ultrasound-guided platelet-rich plasma injections for chronic tendinopathy: A multicenter, retrospective review. Phys Med Rehabil 2013;5:169-175.

30. Lee JJ, Harrison Jr, Boachie-adjei K, Vargas E, Moley PJ. Platelet-rich plasma injections with needle tenotomy for gluteus medius tendinopathy: A registry study with prospective follow-up. Orthop J Sports Med 2016;4: 232-239.

31. Ribeiro AG, Ricioli W Jr, Silva AR, Polesello GC, Guimarães RP. PRP in the treatment of trochanteric syndrome: A pilot study. Acta Ortop Bras 2016;24:208-212.

32. Fitzpatrick J, Bulsara MK, O’Donnell J, McCrory Pr, Zheng MH. The effectiveness of platelet-rich plasma 
injections in gluteal tendinopathy: A randomized, doubleblind controlled trial comparing a single platelet-rich plasma injection with a single corticosteroid injection. Am J Sports Med 2018;46:933-939.

33. van der Worp $H$, Zwerver J, Hamstra M, van den akkerscheek I, Diercks RL. No difference in effectiveness between focused and radial shockwave therapy for treating patellar tendinopathy: A randomized controlled trial. Knee Surg Sports Traumatol Arthrosc 2014;22: 2026-2032.

34. Furia JP, Rompe JD, Maffulli N. Low-energy extracorporeal shock wave therapy as a treatment for greater trochanteric pain syndrome. Am J Sports Med 2009;7: 1806-1813.

35. Naugle KM, Fillingim RB, Riley JL 3rd. A meta-analytic review of the hypoalgesic effects of exercise. J Pain 2012;13:1139-1150.

36. Kosek E, Lundberg L. Segmental and plurisegmental modulation of pressure pain thresholds during static muscle contractions in healthy individuals. Eur J Pain 2003;7:251-258.

37. Hoeger Bement MK, Dicapo J, Rasiarmos R, Hunter SK. Dose response of isometric contractions on pain perception in healthy adults. Med Sci Sports Exerc 2008;40: 1880-1889.

38. Moore D, Semciw AI, Pizzari T. A systematic review and meta-analysis of common therapeutic exercises that generate highest muscle activity in the gluteus medius and gluteus minimus segments. Int J Sports Phys Ther 2020;15:856-881.

39. Kongsgaard M, Qvortrup K, Larsen J, et al. Fibril morphology and tendon mechanical properties in patellar tendinopathy: Effects of heavy slow resistance training. Am J Sports Med 2010;38:749-756.

40. Tortolani PJ, Carbone JJ, Quartararo LG. Greater trochanteric pain syndrome in patients referred to orthopedic spine specialists. Spine J 2002;2:251-254.

41. Howell GE, Biggs RE, Bourne RB. Prevalence of abductor mechanism tears of the hips in patients with osteoarthritis. J Arthroplasty 2001;16:121-123.

42. Mellor R, Bennell K, Grimaldi A, et al. Education plus exercise versus corticosteroid injection use versus a wait and see approach on global outcome and pain from gluteal tendinopathy: Prospective, single blinded, randomised clinical trial. BMJ 2018;361:k1662.

43. Davies JF, Davies DM. Surgical technique for the repair of tears to the gluteus medius and minimus tendons of the hip. J Bone Joint Surg Am 2014;1 1:e11.

44. Stanton MC, Maloney MD, DeHaven KE, Giordano BD. Acute traumatic tear of gluteus medius and minimus tendons in a patient without antecedant peritrochanteric hip pain. Geriatr Orthop Surg Rehabil 2012;3:84.

45. Thaunat M, Noël E, Nové-Josserand L, Murphy CG, Sbiyaa M, Sonnery-Cottet B. Endoscopic management of gluteus medius tendon tears. Sports Med Arthrosc 2016;24: $11-18$.

46. Kocaoglu B, Paksoy AE, Cerciello S, Ollivier M, Seil R, Safran M. Arthroscopic repair of the hip abductor musculotendinous unit: The effect of microfracture on clinical outcomes. Am J Sports Med 2021;49:1570-1577.

47. Saltzman BM, Makhni EC, Stephens JP, Nho SJ. The effect of platelet-rich fibrin matrix at the time of gluteus medius repair: A retrospective comparative study. Arthroscopy 2018;34:832-841. 